\title{
Total Pelvic Exenteration in Leiomyosarcoma - A Case Report
}

\begin{abstract}
Locally advanced high-grade leiomyosarcomas (LMS) with visceral involvement are difficult to manage. Here, we present a case of locally advanced high-grade sarcoma where total pelvic exenteration was undertaken to achieve complete cytoreduction. A 54-year-old lady with a prior history of hysterectomy and bilateral salpingoophorectomy done 8 years back for heavy menstrual bleeding presented to us with an abdomino-pelvic mass. Magnetic resonance imaging of the abdomen suggested large heterogeneous pelvic mass displacing urinary bladder anteriorly and abutting recto-sigmoid posteriorly with no luminal involvement. There was bilateral hydronephrosis. Computed tomography of the thorax revealed no evidence of metastatic disease. A review of previous biopsy and a repeat ultrasound-guided biopsy from the pelvic mass done at our institute suggested spindle-cell neoplasm. In view of pelvis confined disease in a young woman with well-preserved general condition, decision for surgical excision was taken. She underwent total pelvic exenteration with en bloc removal of mass, bladder, and involved portion of recto-sigmoid as the disease was infiltrating both the bladder and the recto-sigmoid. Her postoperative course was turbulent, but she recovered fully and was discharged 20 days after surgery. The operative specimen histopathology revealed high-grade LMS. She received four cycles of adjuvant chemotherapy and thereafter was kept on follow-up. Although she was clinically doing well, her general condition never improved beyond Eastern cooperative oncology Group 2. She also had persistent depressive symptoms. After 14 months of completion of treatment, she recurred with a huge abdominal disease. In view of her general condition and huge disease volume, decision was taken for supportive care only. LMS in advanced stages are difficult to manage. Even after aggressive management with radical exenterative surgery and chemotherapy, we could achieve only temporary control of locally advanced LMS.

Keywords: Chemotherapy, exenteration, extrauterine, leiomyosarcoma
\end{abstract}

\section{Introduction}

Locally advanced high-grade leiomyosarcoma is a difficult disease to manage. Although cytoreductive surgery with complete removal of disease still remains the mainstay of treatment, management in patients with major visceral involvement poses a treatment challenge. The role of neoadjuvant chemotherapy (NACT) in such cases is emerging, but is not established. Here, we present the case of a locally advanced high-grade malignancy where total pelvic exenteration was performed to achieve complete cytoreduction. A literature review for the management of locally advanced disease is undertaken.

\section{Case Report}

A 54-year-old lady presented to us in July 2018 with progressively increasing

This is an open access journal, and articles are distributed under the terms of the Creative Commons Attribution-NonCommercial-ShareAlike 4.0 License, which allows others to remix, tweak, and build upon the work non-commercially, as long as appropriate credit is given and the new creations are licensed under the identical terms.

For reprints contact: WKHLRPMedknow_reprints@wolterskluwer.com abdominal mass for 1 month along with off and on hematuria. There was associated bilateral leg swelling and generalized weakness. Biopsy from the mass done at a previous hospital revealed spindle-cell neoplasm possibly fibroma originating from ovarian remnant. She had also received multiple blood transfusions for severe anemia. She had a history of abdominal hysterectomy and bilateral salpingo-oophorectomy 8 years back for heavy menstrual bleeding. Histopathology confirmed endometriosis and uterine fibroid. There was no history of any significant medical comorbidity or family history of cancer.

She was wheel chair bound due to weakness when she first attended our center. There was mild pallor and Grade 2 pitting edema. Her vital parameters were within the normal range. On abdominal examination, she had firm, fixed, nontender abdominal mass extending from the pelvis

How to cite this article: Sarkar A, Rai S, Singh A, Ghosh J, Bhaumik J. Total pelvic exenteration in leiomyosarcoma - A case report. Indian J Med Paediatr Oncol 2020;41:913-6.

\section{Atanu Sarkar ${ }^{1}$, Shweta Rai ${ }^{1}$, Angad Singh ${ }^{2}$, Joydeep Ghosh ${ }^{3}$, Jaydip Bhaumik ${ }^{1}$}

${ }^{1}$ Department of Gynec Oncology, Tata Medical Center, Kolkata, West Bengal, India, ${ }^{2}$ Department of Pathology, Tata Medical Center, Kolkata, West Bengal, India, ${ }^{3}$ Department of Medical Oncology, Tata Medical Center, Kolkata, West Bengal, India

Submitted: 19-May-2020

Revised: 06-Aug-2020

Accepted: 06-Aug-2020

Published: 31-Dec-2020

Address for correspondence:

Dr. Shweta Rai,

Department Gynec Oncology,

Tata Medical Centre,

14-MAR, New Town, Rajarhat,

Kolkata - 700 160, West Bengal, India.

E-mail: raishweta2005@yahoo. com

Access this article online

Website: www.ijmpo.org

DOI: 10.4103/ijmpo.ijmpo_244_20

Quick Response Code:

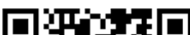


up to the umbilicus. The vaginal vault and rectal mucosa were smooth on pelvic examination. Her complete blood count, coagulation profile, liver, and renal functions were normal. Her CA125 was mildly raised $(68 \mathrm{U} / \mathrm{ml})$, and carcinoembryonic antigen was normal. Lower limbs Doppler ruled out any deep-vein thrombosis. Cystoscopy revealed bladder mucosal involvement in proximity to the urethral orifice. Magnetic resonance imaging suggested $15.5 \mathrm{~cm} \times 13 \mathrm{~cm} \times 15 \mathrm{~cm}$ heterogeneous pelvic mass displacing urinary bladder anteriorly and rectum posteriorly with no luminal involvement. The mass was hyperintense on T2, hypointense on T1 with multiple hypointense septations and irregular loculi with nodular walls with T2 intermediate intensity [Figure 1a and b]. There was bilateral hydronephrosis [Figure 2]. Computed tomography (CT) of the thorax revealed bilateral minimal effusion and no evidence of metastasis. Histopathology review of previously done biopsy done at our institute revealed a spindle-cell tumor with mild atypia, no significant mitotic activity along with the areas of infarction. Immunohistochemistry (IHC) was sparsely positive for desmin and occasional cells showed S-100 expression while negative for beta catenin, CD34, caldesmon, DOG1, CK, c-kit. In view of lack of severe atypia and lack of mitotic activity and noncontributory IHC, a diagnosis of a spindle-cell neoplasm was offered. A repeat ultrasound-guided biopsy was done at our institute which showed similar histopathologic findings. No morphologic features of high-grade pathology were seen [Figure 3a]. In view of her age, possible low-grade tumor and a symptomatic pelvic mass decision were taken for the surgical removal of mass which would entail total pelvic exenteration. Patients and relatives were counseled regarding the procedure, surgical outcome and postoperative morbidities.

She underwent surgery after optimization of her performance status on September 05, 2018. On laparotomy, the pelvic mass was found infiltrating rectosigmoid involving the ileum near the ileocecal junction. It was densely adherent to the bladder with infiltration of the bladder base. She underwent total pelvic exenteration with limited right hemicolectomy, ileocolic and colorectal anastomosis along with ileal conduit and loop ileostomy. Histopathological examination of the resection specimen showed a spindle-cell tumor showing moderate to focally severe atypia, prominent mitotic activity (20-25/10 hpf) along with the large areas of infarction type and scanty areas of tumor type necrosis. IHC showed the tumor cells to be positive for smooth muscle actin, Caldesmon; sparsely positive for Desmin, epithelial membrane antigen while negative for CD117, DOG1, CD34, and S100. The final diagnosis was leiomyosarcoma Fédération Nationale des Centres de Lutte Contre Le Cancer Grade 3 [Figure 3b-d]. Tumor involved the wall of the small bowel and ulcerates the mucosa, invades the serosa of the rectosigmoid and invades the detrusor muscle of the bladder. All resected
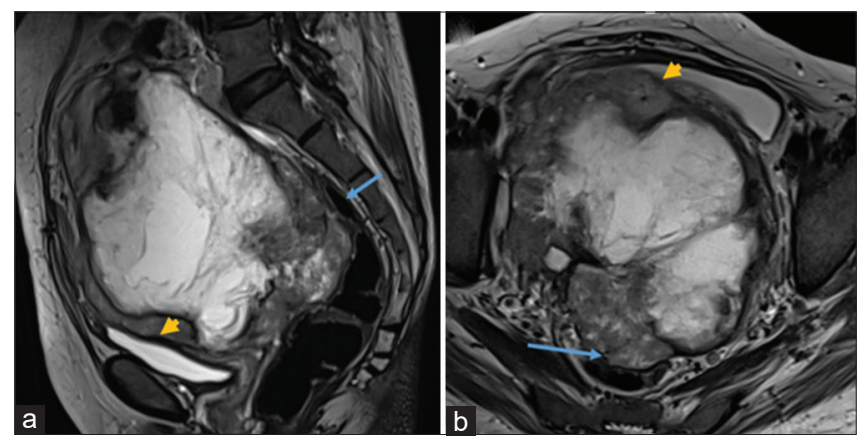

Figure 1: Magnetic resonance imaging of the pelvic mass with loss of planes with bladder (arrowhead) and rectum (arrow) (a) sagittal view (b) axial view

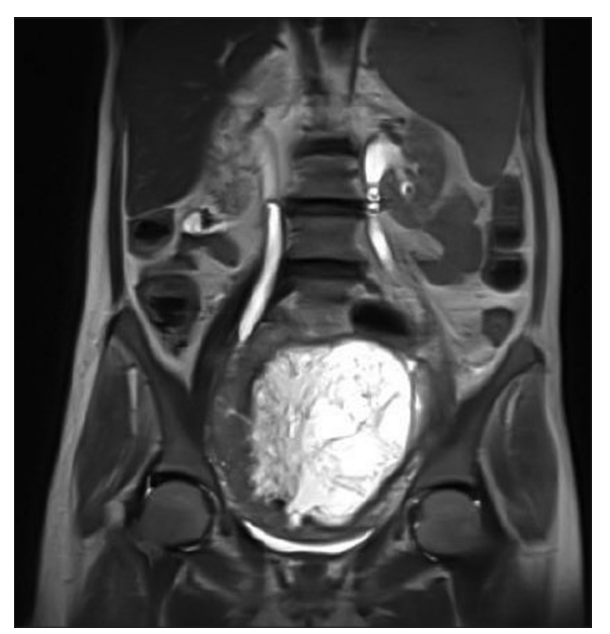

Figure 2: Magnetic resonance imaging abdomen showing bilateral Hydronephrosis due to ureteric encasement

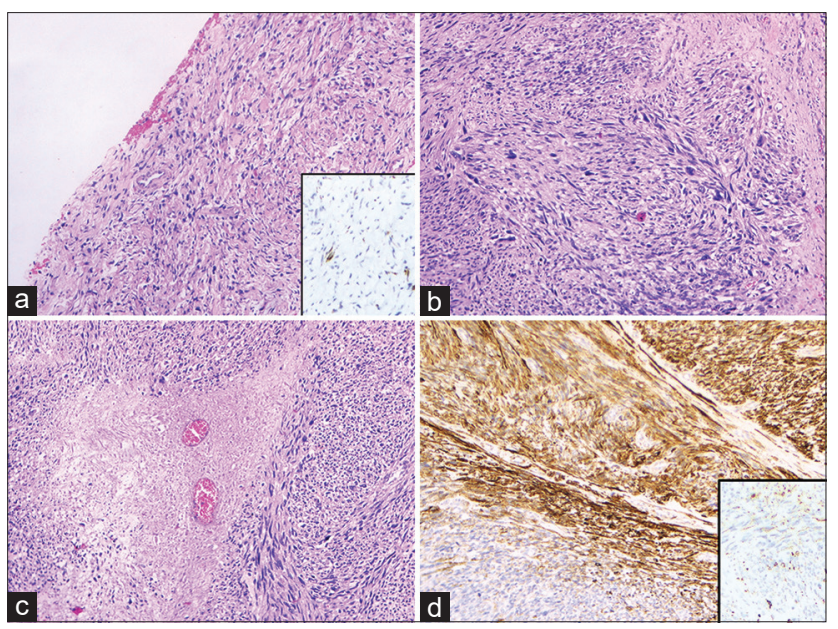

Figure 3: (a) HE section from core biopsy shows spindled cells with minimal atypia. No areas of necrosis or significant mitotic activity seen. Inset shows sparse desmin reactivity in tumor cells $(\times 10)(b)$ HE section from resection specimen shows spindled cells with moderate to focally marked atypia and prominent mitosis $(\times 10)$. Note large pleomorphic cells with hyperchromatic nuclei (c) HE section from resection specimen shows an area of tumor necrosis $(\times 10)(d)$ Immunohistochemistry performed on resection specimen for caldesmon shows strong reactivity in majority of cells. Inset shows sparse desmin positivity $(\times 10)$

margins along with suspected small bowel deposits and omental deposits were negative for malignancy. Her 
postoperative period was turbulent requiring frequent electrolyte corrections and transfusion of multiple blood products, but she recovered well and was allowed home 20 days after her surgery. She received four cycles of adjuvant chemotherapy comprising of gemcitabine and docetaxel. During adjuvant chemotherapy, she was admitted for neutropenic sepsis and managed accordingly. She was seen for follow-up every 3 months. Although there was gradual improvement in her general condition, however, due to sequele of exenterative surgery followed by chemotherapy, it never recovered beyond Eastern Cooperative Oncology Group (ECOG) 2. Furthermore, she required multiple psychological counseling sessions for depressive symptoms. A CT scan done at 6 months of follow-up was normal. Thereafter, she continued with her regular three monthly follow-up till February 2020 when she again presented with abdominal distention. A CT scan was done which showed recurrent disease with $13 \mathrm{~cm} \times 16 \mathrm{~cm} \times 15 \mathrm{~cm}$ abdominal mass involving the sigmoid colon and compressing the left ureter. In view of her poor performance status and a large, un-resectable disease, decision was taken for supportive care only. She succumbed to her disease within a month of her diagnosis of her recurrence.

\section{Discussion}

Spindle-cell neoplasms encompass a wide spectrum of tumors ranging from benign fibroma and leiomyoma to malignant tumors like leiomyosarcoma. Limited sampling of tumor in a small biopsy specimen may be misleading as in our case. ${ }^{[1]}$ According to the WHO classification of tumors of female reproductive organs, leiomyosarcoma is defined as a malignant smooth muscle tumor which commonly shows a spindle cell morphology. Essential histopathologic criteria required for its diagnosis are cytological atypia, mitotic activity, and tumor type necrosis. The former two criteria should usually be seen for diagnosis as it is difficult to distinguish between tumor type necrosis and infarct type necrosis especially in the setting of a small biopsy sample where only limited tissue is available. ${ }^{[2]}$ A study of 100 cases of smooth muscle neoplasms which correlated the core biopsy and the excision tumor histopathological findings found that $68 / 100$ cases showed a grade difference between the two specimens. Among these 68 cases, eight cases were interpreted on biopsy as either "leiomyoma/ smooth muscle tumor of undetermined malignant potential/ smooth muscle tumor: low grade leiomyosarcoma cannot be excluded." All 8 cases on resection were diagnosed with leiomyosarcoma. Although core biopsy is an accurate tool in the diagnosis and grading of soft-tissue tumors, its accuracy is significantly lower in smooth muscle neoplasms. Therefore, the core biopsy findings for smooth muscle neoplasms should be interpreted with caution..$^{[2,3]}$ This highlights the importance of taking the whole clinical, radiological, and pathological picture into account, especially in cases of such voluminous tumors where biopsy samples only a limited portion of tumor.

Retroperitoneal and intraabdominal sarcomas comprise of $15 \%$ of all soft-tissue sarcomas (STS) with leiomyosarcomas (LMS) contributing to nearly half of the total fraction. The most common site of intraabdominal leiomyosarcoma is genitourinary tract, primarily the uterus. ${ }^{[4]}$ In this case, the uterus and ovaries were removed for benign reason around 8 years back. It is possible that in the primary surgical specimen of hysterectomy a small focus of malignancy was missed. The possibility of LMS arising from any other pelvic structure like bladder or bowel muscle cannot be ruled out entirely but is unlikely because of mainly extrinsic involvement of these structures by the mass and also that an uterine origin is by far the most common.

Most of the tumor presents with asymptomatic abdomino-pelvic mass with bowel, bladder, and vascular pressure symptoms in the $5^{\text {th }}$ decade like our patient. ${ }^{[5]}$ Complete resection of all visible tumor is the corner stone of management. The surgery should aim to achieve macroscopically complete resection with en-bloc removal of tumor along with all involved structures. Best chance of resection with curative intend is at the time of primary presentation. ${ }^{[6]}$ The treatment decision is generally straightforward in case of disease confined to the uterus, and there are no extra-uterine disease to necessitate multi-visceral resections. In a widely metastatic disease, management with chemotherapy with palliative intent is well accepted. However, management in a locally advanced or abdomen restricted disease like ours entailing multi-visceral resections and total exenterating is a difficult decision to take. Preoperatively, our decision for exenterative surgery was not difficult as the diagnosis was of a low-grade spindle cell neoplasm. Unfortunately, the histopathology report changed to high-grade sarcoma in the postoperative specimen. This definitely had an impact on the final prognosis of the disease. Could the decision to go for upfront surgery be different if we knew about the high-grade nature of the disease is a point to ponder. Leitao et al. did a retrospective single institution review of high-grade LMS with extrauterine disease at initial presentation. Out of 96 women, intraperitoneal disease was seen in $48(50 \%)$ and extraperitoneal in the other $48(50 \%)$ women. Complete gross resection was possible in $49 \%$. Eighty percent of all women received adjuvant treatment, mostly doxorubicin-based chemotherapy. Ninety-seven percent women recurred at some point. The median progression-free survival was significantly longer in those with complete cytoreduction versus those with any residual disease (14.2 months vs. 6.8 months, $P=0.002$ ). The overall survival was not found to have significant difference. ${ }^{[7]}$

The role of NACT in such advanced cases has been investigated with the expectation to enhance the operability 
and make conservative surgery more likely. Randomised trials to date have been done broadly including STS of all sites. $^{[8]}$ These are heterogeneous trials, including patients with varying tumor size, grade, histological subtype, and anatomical locations. Extrapolation of the results of these trials for aggressive intraabdominal LMS should be done with caution for the reasons mentioned and also as these tumors are relatively chemo-resistant compared to other STS. A randomised trial by Gronchi et al. analyzed the outcome of histotype-tailored NACT compared to standard five cycles of perioperative chemotherapy in STS. ${ }^{[9]}$ However, no definite conclusion could be drawn regarding their efficacy for these tumors. A randomized trial analyzing the role of NACT in retroperitoneal leiomyosarcoma and fibrosarcoma is at present in the inception phase. We may hope the results of this trial to give some definitive answers to our queries. ${ }^{[10]}$

Postoperatively, our patient was treated with adjuvant four cycles of gemcitabine and docetaxel. She had chemotherapy-related side effects from which she recovered to complete her chemotherapy. However, her functional status never improved above ECOG 2 and that too associated with frequent episodes of depression.

Our patient had 14 months of disease-free survival. She was not fit for any chemotherapy in the recurrent setting due to her poor general condition. Some studies have reported the role of hormone therapy in cases of receptor positive of high-grade LMS in recurrent/inoperable settings. However, they do not seem to offer any benefit in cases of high volume recurrences.

We had chosen to keep her on only clinical follow-up after first normal follow-up scan at 6 months. Could more frequent CT scans have helped us pick up the recurrence earlier is a matter of debate. It is difficult to agree to a follow-up plan with more frequent CT scans as the tumour can grow really fast in a recurrent setting. A normal CT scan on a patient who is clinically well will not guarantee of not having a large recurrence only a few weeks later. Even if we had identified the disease a few weeks earlier when the tumour volume was less, venturing into another complicated surgery or chemotherapy might not have been justified given her performance status.

\section{Conclusion}

Management in advanced leiomyosarcoma has to be individualized depending on patient's performance status, age, resectability, and acceptance of postoperative morbidities. Surgery remains the mainstay of treatment though the overall outcome in these setting remains to be poor. The role of NACT in these tumors can be explored though the evidence for same is very meager.
Informed consent had been taken from the patient.

\section{Declaration of patient consent}

The authors certify that they have obtained all appropriate patient consent forms. In the form, the patient has given her consent for her images and other clinical information to be reported in the journal. The patient understand that name and initials will not be published and due efforts will be made to conceal identity, but anonymity cannot be guaranteed.

\section{Financial support and sponsorship}

Nil.

\section{Conflicts of interest}

There are no conflicts of interest.

\section{References}

1. Abeler VM, Røyne O, Thoresen S, Danielsen HE, Nesland JM, Kristensen GB. Uterine sarcomas in Norway. A histopathological and prognostic survey of a total population from 1970 to 2000 including 419 patients. Histopathology 2009;54:355-64.

2. Kurman RJ, Carcangiu ML, Herrington CS, Young RH, editors. WHO Classification of Tumours of Female Reproductive Organs. $4^{\text {th }}$ ed. Lyon: IARC; 2014. p. 135-48.

3. Schneider N, Strauss DC, Smith MJ, Miah AB, Zaidi S, Benson $\mathrm{C}$, et al. The Adequacy of core biopsy in the assessment of smooth muscle neoplasms of soft tissues: Implications for treatment and prognosis. Am J Surg Pathol 2017;41:923-31.

4. DeVita VT Jr., Lawrence TS, Rosenberg SA. Devita, Hellman, and Rosenberg's Cancer: Principles \& Practice of Oncology. $11^{\text {th }}$ ed. Place of Publication Lippincott Williams \& Wilkins (LWW); 2018.

5. Priya BS, Kathiresan K, Anandan H. Retroperitoneal soft tissue sarcoma: An analysis of surgical and adjuvant chemotherapy. Int J Sci Stud 2017;4:154-7.

6. Dumitra S, Gronchi A. The diagnosis and management of retroperitoneal sarcoma. Oncol J 2018;32:464-9.

7. Leitao MM Jr., Zivanovic O, Chi DS, Hensley ML, O'Cearbhaill R, Soslow RA, et al. Surgical cytoreduction in patients with metastatic uterine leiomyosarcoma at the time of initial diagnosis. Gynecol Oncol 2012;125:409-13.

8. Gortzak E, Azzarelli A, Buesa J, Bramwell VH, van Coevorden F, van Geel AN, et al. A randomised phase II study on neo-adjuvant chemotherapy for 'high-risk' adult soft-tissue sarcoma. Eur J Cancer 2001;37:1096-103.

9. Gronchi A, Ferrari S, Qsuagliuolo V, Broto JM, Pousa AL, Grigani G, et al. Histotype-tailored neoadjuvant chemotherapy versus standard chemotherapy in patients with high-risk soft-tissue sarcomas (ISG-STS 1001): An international, open-label, randomised, controlled, phase 3, multicentre trial. Lancet Oncol 2017;18: 812-22.

10. Surgery With or Without Neoadjuvant Chemotherapy in High Risk Retroperitoneal Sarcoma (STRASS2). ClinicalTrials.gov Identifier: NCT04031677. Available from: https://clinicaltrials. gov/ct2/show/NCT04031677. [Last update on 2020 May 14]. 